Trauma Berufskrankh 2010 • 12 [Suppl 4]:387-396 DOI 10.1007/s10039-010-1673-0

Online publiziert: 7. Oktober 2010

(c) Springer-Verlag 2010

T. Mückley $\cdot$ K. Klos

Klinik für Unfall-, Hand- und Wiederherstellungschirurgie,

Friedrich-Schiller-Universität Jena

\title{
Arthrodesen am Sprunggelenk und Rückfuß
}

\section{Diagnostik}

\section{Anamnese und Klinik}

Neben der Anamneseerhebung ist die klinische Untersuchung die Grundlage der Diagnostik. Es werden der Durchblutungsstatus, die Hauttrophik und die Beweglichkeit der angrenzenden Gelenke ermittelt. Hierbei werden Fehlstellungen und Bewegungseinschränkungen erfasst. Bei der zunehmenden Patientenzahl mit Diabetes mellitus bzw. pAVK (periphere arterielle Verschlusskrankheit) ist der differenzierten Abklärung von evtl. vorliegenden Durchblutungsstörungen besondere Aufmerksamkeit zu widmen.

\section{Bildgebende Diagnostik}

Standardröntgenuntersuchungen sind die Sprunggelenkaufnahme in 2 Ebenen sowie - zur Betrachtung der Rückfußachse - die Salzmann-Aufnahme im Stand (• Abb. 1a-c). Hiermit können ggf. Instabilitäten detektiert werden, des Weiteren gibt diese Aufnahme Aufschluss über Achsfehlstellungen im Rückfußbereich unter Belastung.

Ergänzend führen wir bei komplizierteren Fällen regelmäßig eine CT-Untersuchung (CT: Computertomographie) mit $2 \mathrm{D}$ - und $3 \mathrm{D}$-Rekonstruktion durch (- Abb. 1b,c, - Abb. 2a,b). Hiermit können Knochendefekte exakter detektiert und die Ausprägung der Deformität besser analysiert werden.

Eine Infiltration des betroffenen $\mathrm{Ge}$ lenks unter Durchleuchtungskontrolle mit Lokalanästhetikum kann in der Abklärung der betroffenen Gelenke zusätzliche Informationen liefern (• Abb. 3). Grundsätzlich ist es anzustreben, so wenige Gelenke wie notwendig zu fusionieren. Insofern können angrenzende Gelenke, die in der Beweglichkeit eingeschränkt sind, aber durch Infiltration das Beschwerdebild des Patienten nicht verändern, von der Arthrodese ausgenommen werden.

Im Zweifelsfall kann die SPECT („single photon emission computed tomography") entscheidende Zusatzinformationen liefern [14].

Die Magnetresonanztomographie (MRT) ist in Einzelfällen, speziell zur Beurteilung der Durchblutung des Talus, hilfreich ( $\bullet$ Abb. 2c). Bei partiellen Talusnekrosen kann mit ihrer Hilfe besser zwischen einer notwendigen isolierten tibiotalaren und einer tibiotalokalkanearen Arthrodese (TTCA) differenziert werden.

Das PET-CT (PET: Positronenemissionstomographie) kann in Einzelfällen hilfreich sein, um bei abgelaufenen Infektionen die Aktivität des Infektgeschehens einzuschätzen.

\section{Arthrodese am Sprunggelenk}

\section{Grundprinzip}

Wie an anderen Körperregionen auch ist die rechtzeitige Indikationsstellung entscheidend. Die angrenzenden Gelenke sollten möglichst intakt sein und eine gute Beweglichkeit aufweisen. 


\section{Sprunggelenk und Fuß}
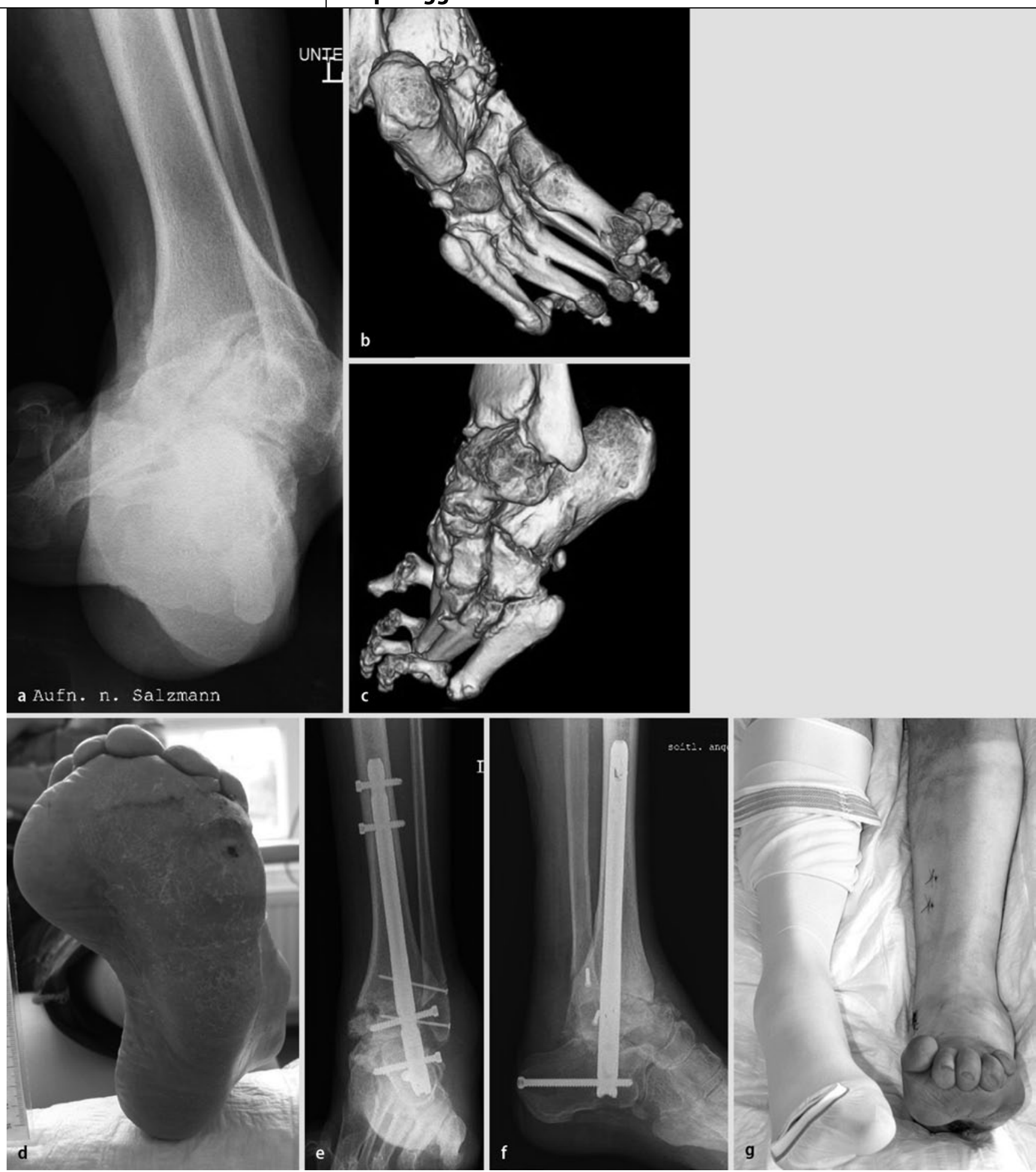

Abb. 1 \ Massive Klumpfußdeformität, a Salzmann-Aufnahme, b,c entsprechende 3D-CT-Rekonstruktionen, $\mathbf{d}-\mathbf{g}$ Korrekturpotenzial einer tibiotalokalkanearen Arthrodese, $\mathbf{d}$ präoperative Fotografie, e,f postoperative Röntgenaufnahmen, $\mathbf{g}$ postoperative Fotodokumentation

Bei den Arthrodeseverfahren im Rückfußbereich werden die Knorpelflächen vollständig reseziert. Nach der Präparation sollen möglichst vitale, gut blutende Knochenflächen vorliegen.
Knöcherne Defekte sowie strukturell reduzierte Knochenflächen können ggf. mit autologer Spongiosaplastik aufgefüllt bzw. augmentiert werden. Wir führen bei nahezu allen Sprunggelenkarthrodesen eine Spongiosaplastik durch $[7,8]$.
Bei Korrekturarthrodesen kann Spongiosa aus den Knochenresektaten oder bei transfibulären Zugängen mit Außenknöchelosteotomie aus der resezierten $\mathrm{Ge}$ lenkfläche des Außenknöchels gewonnen werden. Erfahrungsgemäß ist diese 
Spongiosa nicht mit der gewohnten guten Qualität der Beckenkammspongiosa vergleichbar.

Die subchondrale Knochenschicht der Arthrodeseflächen muss angefrischt werden, hierzu sind Anbohrungen der Knochenfläche mit einem 2,5-mm-Bohrer oder ein Aufmeißeln der subchondralen Knochenschicht mit einem schmalen Meißel möglich.

\section{Einstellung}

Sie erfolgt hinsichtlich

- Dorsalextension/Plantarflexion in Neutralstellung,

- Rückfußvalgusstellung in einem Winkel von $5^{\circ}$ und

- Außenrotation des Fußes in einem Winkel von etwa $5^{-10^{\circ}}$.

Dabei ist der Vergleich mit der Gegenseite hilfreich. Eine Reorientierung des Talus unter die Tibiaachse ist anzustreben und kann am besten im seitlichen Bildwandlerbild beurteilt werden. Wenn der Processus fibularis des Talus in Verlängerung der anatomischen Tibiaachse zu liegen kommt, ist eine korrekte anatomische Platzierung des Talus unter die Tibiaachse gewährleistet. Bei Verkürzungen im Rückfußbereich durch z. B. Sinterungen des Talus ist ein Impingement der $\mathrm{Au}$ ßenknöchelspitze an der lateralen Wand des Kalkaneus bzw. der Peronäalsehnen möglich und zu berücksichtigen. Gegebenenfalls ist in diesen Fällen eine Verkürzungsosteotomie der Fibula sinnvoll.

Neben den bereits genannten Aspekten der korrekten Einstellung der Rückfußarthrodese sind die anatomische Wiederherstellung der Cyma-Linie und die möglichst korrekte Rekonstruktion der Rückfußhöhe zu beachten. Eine Verminderung der Rückfußhöhe von 0,5-1,o cm wird von uns in der Regel toleriert, größere Höhenverluste sollten möglichst vermieden werden. In diesen Fällen sind, wenn möglich, Knochen rekonstruierende Verfahren anzuwenden (autologe Spongiosa, allogene Spongiosablöcke).

Inkorrekte Rückfußeinstellungen im Sinne von Varuseinstellungen sind dringend zu vermeiden. Bei Varusfehlstellungen im Rückfußbereich verliert die bei physiologischer Rückfußachse paral-
Trauma Berufskrankh 2010 - 12 [Suppl 4]:387-396 DOI 10.1007/s10039-010-1673-0

C) Springer-Verlag 2010

T. Mückley $\cdot$ K. Klos

Arthrodesen am Sprunggelenk und Rückfuß

\section{Zusammenfassung}

Indikationen zur Arthrodese des Sprunggelenks sind posttraumatische Folgezustände, rheumatoide Arthritis, Gelenkinfekt, diabetische Osteoarthropathie, fehlgeschlagene Endoprothetik, ideopathische Arthrosen und neuromuskuläre Fehlstellungen. Es werden Arthrodesen des oberen (tibiotalar) und des unteren Sprunggelenks (talokalkanear) sowie des gesamten Rückfußes (tibiotalokalkanear) unterschieden. Anamnese und klinische Untersuchung sind die Grundlage der Diagnostik, ergänzt werden sie durch bildgebende Verfahren. Grundprinzip der Arthrodese am Sprunggelenk ist die rechtzeitige Indikations-

stellung. Die Gelenkflächenpräparation kann konventionell offen oder arthroskopisch erfolgen, wobei Ersteres das Standardverfahren darstellt. Als Osteosynthesetechniken kommen der Fixateur externe, Schrauben-, Nagelund Plattentechniken zur Anwendung. Die korrekte Einstellung des Rückfußes ist für das funktionelle Outcome entscheidend.

\section{Schlüsselwörter \\ Sprunggelenk · Arthrodese . \\ Gelenkflächenpräparation · Orthopädische \\ Fixationstechniken · Rückfußeinstellung}

\section{Arthrodesis of the ankle and hindfoot}

\section{Abstract}

Indications for arthrodesis of the ankle include post-traumatic sequelae, rheumatoid arthritis, joint infection, diabetic osteoarthropathy, failed arthroplasty, idiopathic arthritis and neuromuscular misalignment. Distinction is made between arthrodesis of the upper ankle (tibiotalar), the lower ankle (talocalcaneal) and the entire hindfoot (tibiotalocalcaneal). Patient history and clinical examination form the basis of diagnosis and may be complemented by imaging techniques. The main principle of ankle arthrodesis is prompt establishment of the indication. Pre- paring the joint surface can be performed either by conventional open procedure or arthroscopically, whereby the former is considered the standard procedure. Osteosynthesis techniques include external fixation as well as screw, plate and nail techniques. Correct alignment of the hindfoot is essential for a functional outcome.

\section{Keywords}

Ankle joint · Arthrodesis · Joint surface preparation - Orthopaedic fixation devices . Hindfoot alignment 


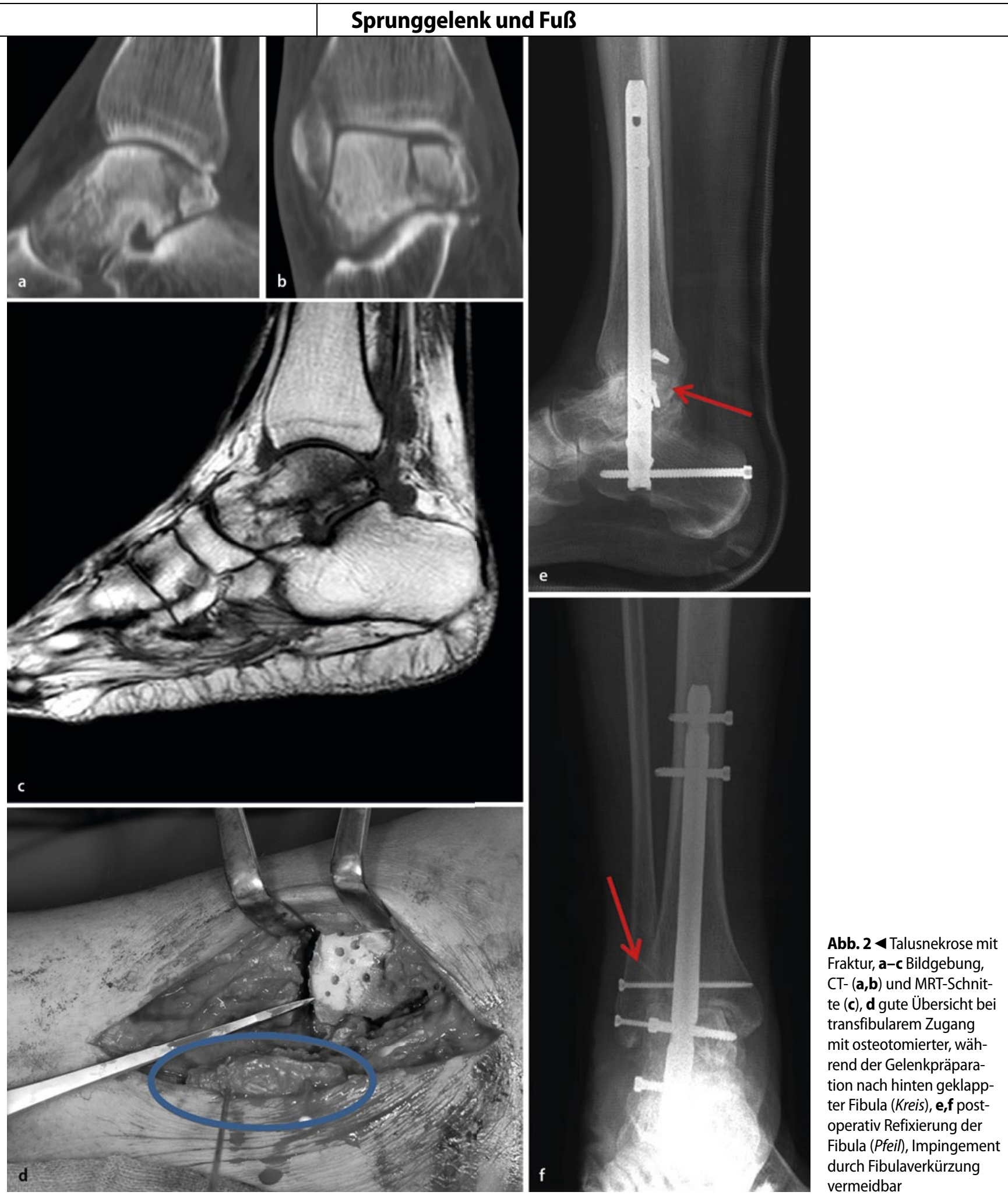

lele Ausrichtung der talonavikularen und kalkaneokuboidalen Gelenkachse ihre Parallelität (• Abb. 4). Dadurch wird die Beweglichkeit in der Chopart-Gelenklinie bei Varusfehlstellungen im Rückfuß erheblich eingeschränkt [4]. Das Gangbild mit eingeschränkter Abrollfunktion führt dann zu einer Überlastung der lateralen
Säule mit Hyperkeratose der Fußsohle und entsprechender Beschwerdesymptomatik. Regelmäßig müssen varische Rückfußfehlstellungen aufgrund der Beschwerdesymptomatik der Patienten korrigiert werden ( $\bullet$ Abb. 5).

\section{Gelenkflächenpräparation}

Sie kann für die Arthrodese des Sprunggelenks in konventioneller offener Technik oder auch arthroskopisch erfolgen. 
Abb. $3 \longrightarrow$ Testinfiltration (Pfeil Nadel) in das untere Sprunggelenk bei stattgehabter Arthrodese des oberen Sprunggelenks

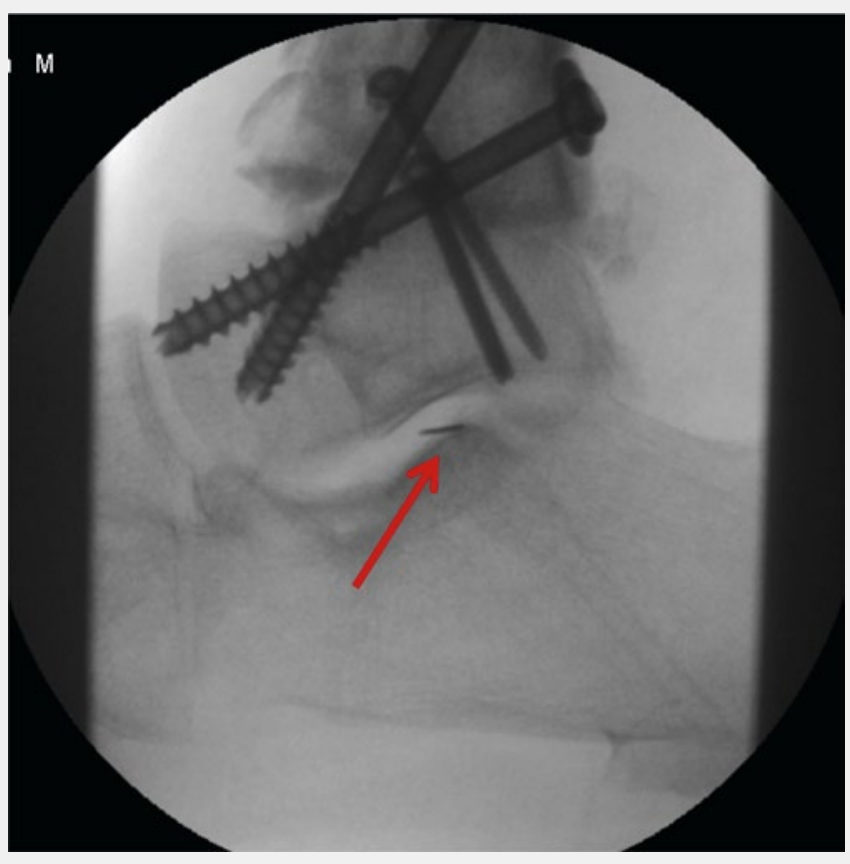

Abb. 4 a Physiologische Rückfußachse mit, b varische Einstellung ohne Parallelität der talonavikularen (1) und kalkaneokuboidalen (2) Gelenkachse

Abb. 5 $>$ In Varusfehlstellung eingestellte tibiotalokalkaneare Arthrodese (a) mit fast ausschließlicher Belastung der lateralen Säule (b) mit entsprechender Hyperkeratose der Fußsohle (c), nötige Korrektur (d)
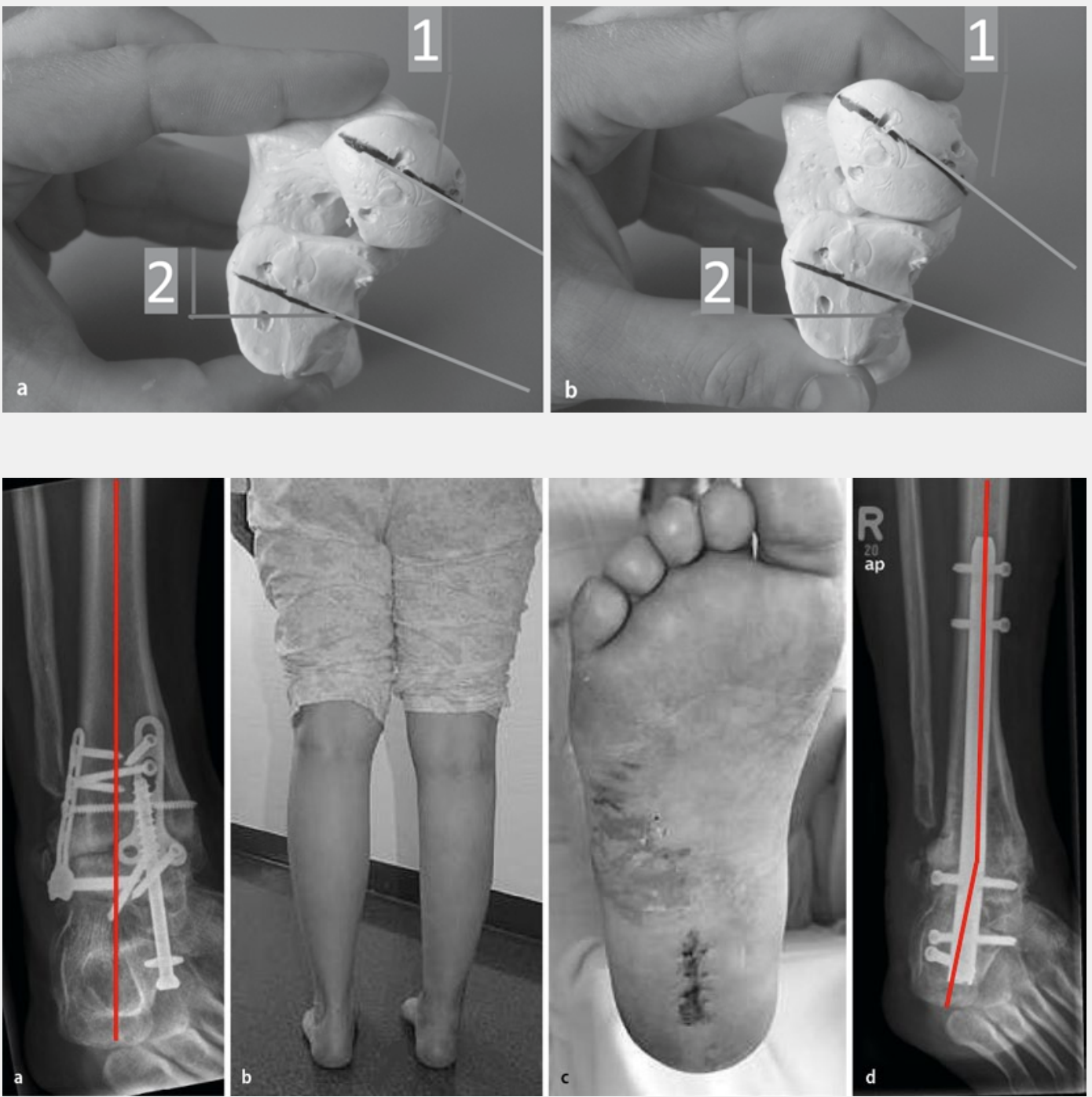


\section{Sprunggelenk und Fuß}
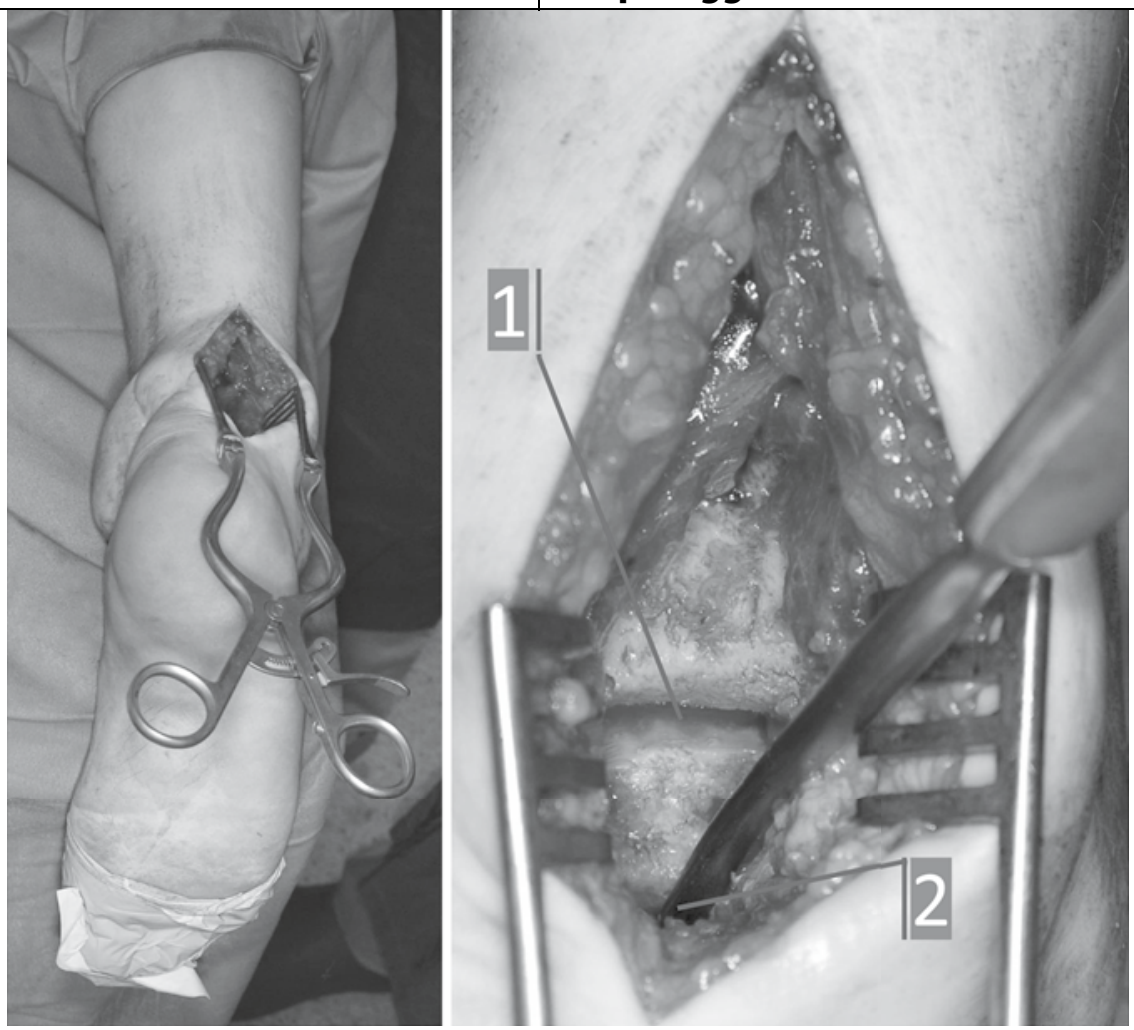

Abb. $6 \Delta$ Dorsaler paraachillärer lateraler Zugang mit Einsicht in oberes (1) und unteres (2) Sprunggelenk

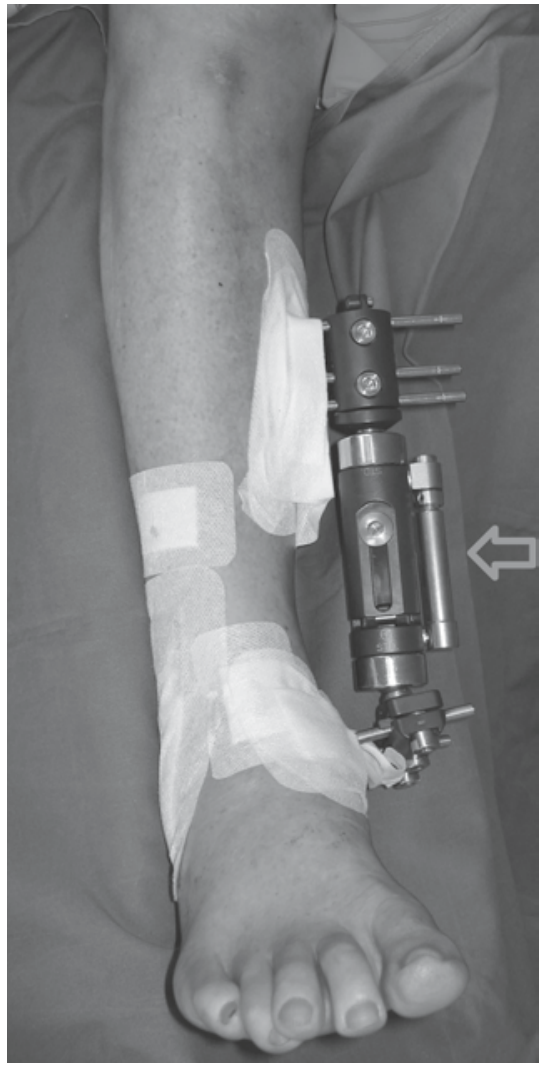

Abb. 7 A Fixateur externe mit Kompressionsvorrichtung (Pfeil) zur OSG-Arthrodese

\section{Arthroskopie}

Es sind eine Traktionsvorrichtung, ein Standardarthroskop, moderne Flüssigkeitspumpensysteme, ein Weichteil-Shaver und Knochenfräseraufsätze erforderlich. Vorteile der arthroskopischen Gelenkflächenpräparation sind eine schnellere Durchbauungszeit und höhere Fusionsraten $[5,6]$. Der Blutverlust ist im Vergleich zum offenen Vorgehen geringer.

Eine Verminderung der Schmerzsymptomatik durch die arthroskopische im Vergleich zur offenen Technik kann durch die Autoren nicht bestätigt werden. Bei sehr kontrakten Gelenken kann die arthroskopische Präparation des Gelenks unmöglich sein. Nachteile der arthroskopischen Technik am OSG sind weiterhin die schwierige Präparation im vorderen Gelenkrecessus und die aufwändige Darstellung der Gelenkflächen. Kontraindikation stellen hochgradige Achsfehlstellungen, größere Knochendefekte und ein mechanisch nicht aufdehnbares Gelenk dar.

\section{Konventionelle offene Technik}

Sie stellt das Standardverfahren für die Arthrodese der Sprunggelenke dar.
Zum OSG kann ein ventraler Zugang mit der Leitschiene der Sehne des M. tibialis anterior gewählt werden.

Für die tibiotalokalkaneare Arthrodese ist ein transfibularer Zugang mit Osteotomie des Außenknöchels gut geeignet. Hier können die tibiotalare und die talokalkaneare Gelenkfläche gut eingesehen und präpariert, entsprechende Fehlstellungen ausgeglichen und Spongiosa angelagert werden. Nach Abschluss der Arthrodese kann der Außenknöchel nach Resektion der Gelenkfläche zurückgeklappt und mit 2 Kleinfragmentschrauben in der Tibia bzw. dem Talus refixiert werden (• Abb. 2d-f). Dadurch können die Peritonäalsehnen in ihrer Leitschiene dorsal des Außenknöchels sowie das Periost in den dorsalen Außenknöchelanteilen belassen werden, um somit die Durchblutungssituation im Bereich der Arthrodese zusätzlich zu verbessern.

Die tibiotalokalkaneare Arthrodese kann auch in Bauchlage über einen dorsalen paraachillären lateralen Zugang präpariert werden ( $\bullet$ Abb. 6), über welchen ggf. eine entsprechende Achillessehnenverlängerung durchführbar ist.

Korrekturen im Valgus- und Varussinne sind in den Händen der Autoren über einen rein dorsalen Zugang schwieriger zu bewerkstelligen als über einen transfibularen Zugang.

Letztendlich sind die Zugänge für jeden Patienten individuell zu planen und von den vorliegenden Weichteilbedingungen abhängig zu machen. Ausgeprägte langjährige Valgusstellungen des Rückfußes sollten bei Korrekturoperationen mit Aufrichtung des Valgus und Reorientierung des Fußes unter die Tibiaachse möglichst nicht über einen lateralen $\mathrm{Zu}$ gang operiert werden. Das Stretching der langjährig verkürzten Hautweichteile lateralseitig führt beim Aufrichten des Rückfußes zu Durchblutungsstörungen im $\mathrm{Zu}$ gangsbereich und regelmäßig zu Wundheilungsstörungen. Hier sind alternative Zugänge, $\mathrm{z}$. B. dorsalseitig, zu bevorzugen.

Eine Knochen sparende Resektion der Knorpel tragenden Flächen ist anzustreben. Insofern werden bei korrekter Rückfußachse nur die Gelenkoberfläche im Sinne einer In-situ-Arthrodese entfernt und die subchondralen Knochen- 
schichten mittels Bohrung oder Meißel angefrischt. Fehlstellungen erfordern eine entsprechende Keilresektion.

\section{Osteosyntheseverfahren}

Implantate für Arthrodesen am Rückfuß sollten eine möglichst stabile Fixierung, die zum einen eine sichere knöcherne Durchbauung und zum anderen eine frühere Mobilisation der angrenzenden Gelenke ermöglicht, mit Kompression der aneinander gelagerten Knochenflächen gewährleisten.

Es kommen unterschiedliche Osteosynthesetechniken zur Anwendung.

\section{Fixateur externe}

Er ist nach wie vor der Standard für Infektsituationen bzw. Fälle mit kritischen Weichteilschäden. Nachteile sind die hohe Rate an Pininfektionen, der verminderte Tragekomfort, die lange Tragedauer, die höhere Pseudarthroserate im Vergleich zu den internen Osteosyntheseverfahren und die Beeinträchtigung der angrenzenden Gelenke.

Grundsätzlich sollten die Fixateur-externe-Montagen eine Kompressionsvorrichtung enthalten, um die Knochenflächen aufeinanderpressen zu können.

- Abb. 7 zeigt eine medial angebrachte Fixateur-externe-Montage für die tibiotalare Arthrodese mit Kompressionsmöglichkeit. Für isolierte tibiotalare Arthrodesen sollte mit dem Fixateur externe das untere Sprunggelenk geschont werden. Für tibiotalokalkaneare Arthrodesen mit Fixateur externe ist der Ringfixateur mit Fußplatte zu bevorzugen.

\section{Schraubentechniken}

Sie stellen für die primäre tibiotalare Arthrodese sowie die primäre subtalare (talokalkaneare) Arthrodese das Standardverfahren dar. Zur Abstützung der Schraubenköpfe benötigt man eine entsprechend gute Knochenqualität in diesem Bereich. Insofern kommen bei Revisionsarthrodesen bzw. bei Arthrodesen nach Pilonfrakturen mit entsprechend reduzierter Knochenqualität im Bereich der distalen Tibia andere Methoden zum Einsatz.

Für die tibiotalare Arthrodese verwenden wir in der Regel eine 3-Schrauben-Technik, ggf. können eine 4. Schrau-
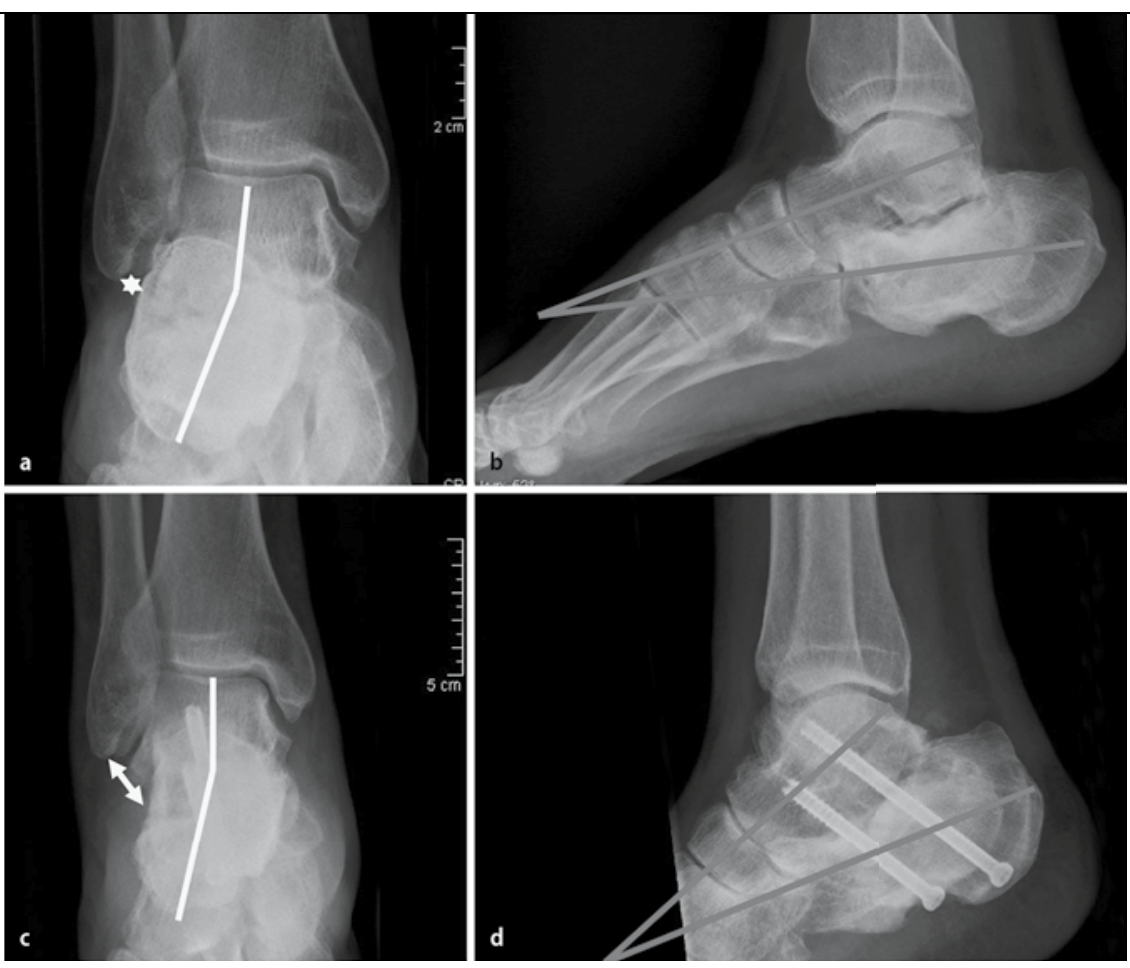

Abb. 8 A USG-Arthrodese mit korrigierendem trikortikalem Beckenkamminterponat, a,b prä-, c,d postoperativ, a,c Stern, Pfeil Aufhebung des lateralen Impingements, b,d Rekonstruktion des talokalkanearen Winkels

be von der Fibula horizontal in den Talus gebohrt bzw. eine 5. Schraube suprasyndesmal von der Fibula in die distale Tibia eingebracht werden. Es können Großfragmentschrauben bzw. kanülierte 6,o- bzw. 7,3-mm-Schrauben verwendet werden.

Für die subtalare Schraubenarthrodese, auch häufig als Korrekturarthrodese nach fehlverheilten Kalkaneusfrakturen, verwenden wir nach Interposition korrigierender trikortikaler Beckenkammspäne 2 kanülierte Schrauben in paralleler Ausrichtung vom Fersenbein retrograd in den Talus zielend ( $\bullet$ Abb. 8). Ebenso kommen Großfragmentschrauben bzw. kanülierte 6,o- bzw. 7,3-mm-Schrauben zur Anwendung.

\section{Nageltechniken}

Sie finden bei der antegraden tibiotalaren Arthrodese ( $\bullet$ Abb. 9) und der retrograden tibiotalokalkanearen Arthrodese Verwendung (• Abb. 2d-f, $\bullet$ Abb. 5).

Antegrade Arthrodese. Für die antegrade tibiotalare Arthrodese sind spezielle Verriegelungsnägel erforderlich. Der distale T2-Tibiamarknagel (Fa. Stryker) ist für die spezielle Indikation der antegraden OSG-Arthrodese zugelassen und weist eine entsprechende Verriegelungskonfiguration an der Nagelspitze auf. Hier können 2 in einem Winkel von $90^{\circ}$ zueinander stehende Verriegelungsschrauben in einem Abstand von insgesamt $2 \mathrm{~cm}$ im Talus platziert werden. Insofern ist im Bereich der Nagelspitze eine Talusmindesthöhe von $2 \mathrm{~cm}$ erforderlich. Des Weiteren bietet der Nagel die Möglichkeit der Kompressionsausübung.

Nägel mit winkelstabilen Verriegelungsmechanismen sind aktuell für die Indikation der antegraden OSG-Arthrodese noch nicht zugelassen. Die Technik der Instrumentierung ist anspruchsvoll, und es besteht der Nachteil des knienahen Zuganges. Postoperativ kann es somit zur Komplikation des knienahen Schmerzes nach Tibiamarknagelung kommen. Des Weiteren ist bei einer postoperativen Infektion die Ausbreitung im eröffneten und instrumentierten Markkanal möglich.

Eine ideale Indikation für die antegrade Nagelarthrodese des oberen Sprunggelenks sind längerstreckige posttraumatische Knochendefekte bzw. noch nicht vollständig konsolidierte Frakturkomponenten im Pilonbereich. Auch parallel vorliegende Pseudarthrosen im Bereich der distalen Tibia mit Destruktionen des 


\section{Sprunggelenk und Fuß}

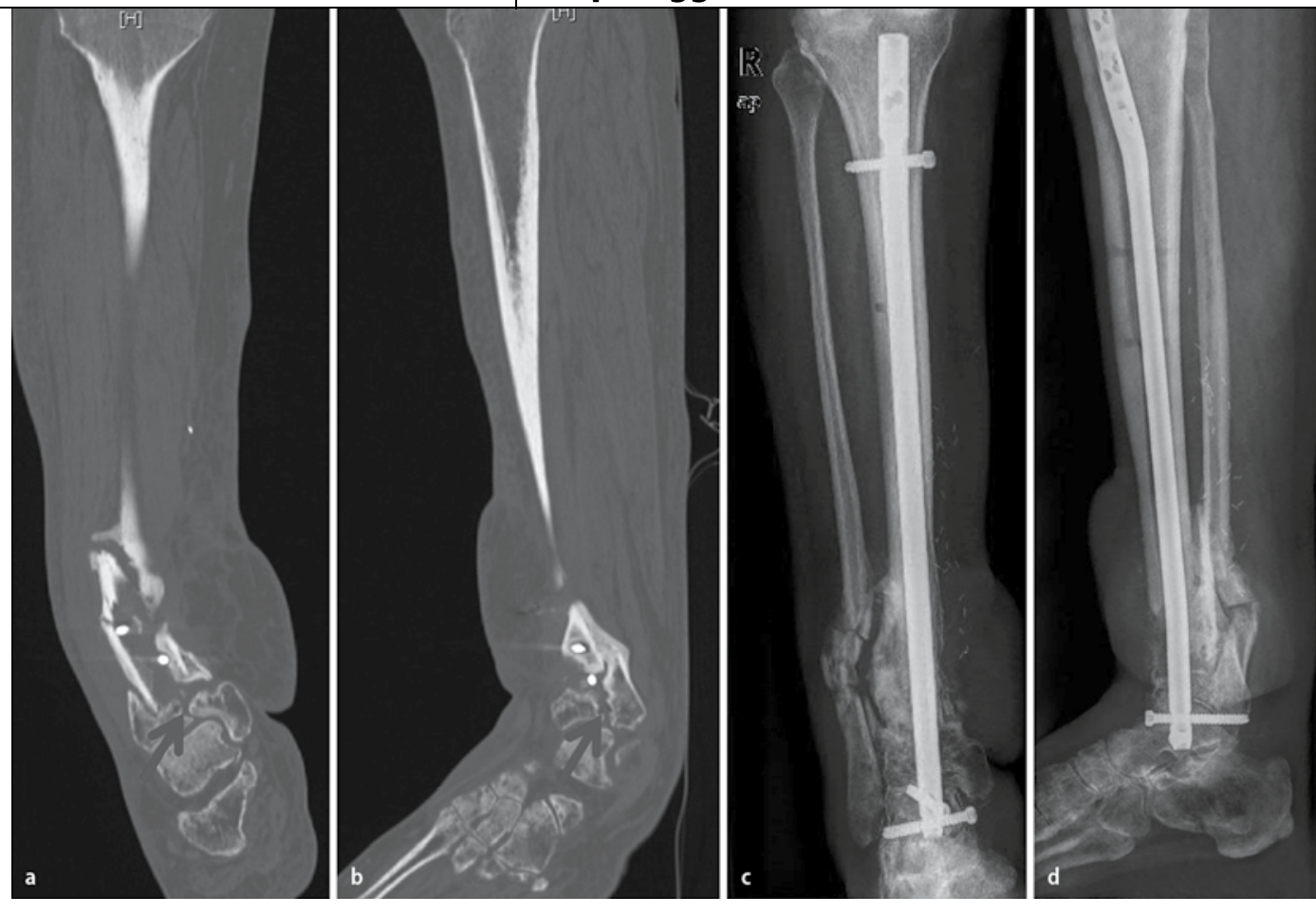

Abb. $9<$ Nach Meinung der Autoren ideale Indikation für OSG-Arthrodese mittels antegradem $\mathrm{Na}$ gel, $\mathbf{a}, \mathbf{b}$ exemplarische CTSchnitte mit Pseudarthrose und massiver Gelenkflächendestruktion (Pfeile), c, d postoperative Röntgenbilder
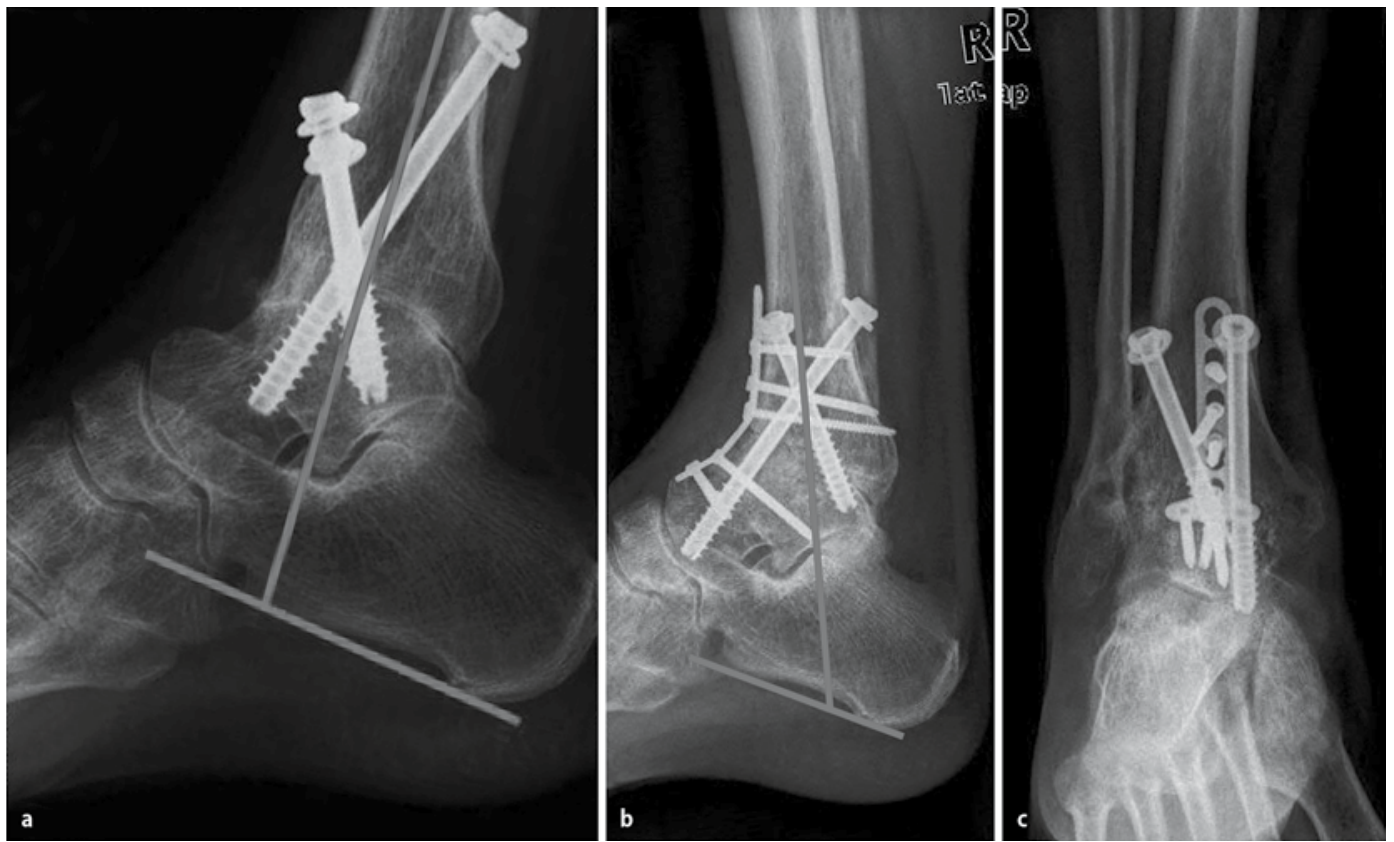

Abb. $10<$ Korrektur einer in Spitzfußfehlstellung eingestellten OSG-Arthrodese (a) mittels Schraubentechnik $(\mathbf{b}, \mathbf{c})$

oberen Sprunggelenks können mit der antegraden Nageltechnik gleichzeitig überbrückt werden (ब Abb. 9). In diesen Fällen bietet der Nagel biomechanisch Vorteile gegenüber den Schraubentechniken und benötigt im Vergleich zu den Plattentechniken kleinere Weichteilinzisionen [9, 10,11 .

Retrograde Arthrodese. Die retrograde tibiotalokalkaneare Marknagelarthrodese erfreut sich in den letzten Jahren zuneh- mender Beliebtheit. Zum einen bieten die meisten Marknagelsysteme für diese Indikation hohe biomechanische Primärsteifigkeiten und zum anderen sind nur limitierte Inzisionen für diese Osteosynthesetechnik erforderlich [13, 15]. Es dürfen allerdings keine Einschränkungen in Bezug auf die Gelenkflächenpräparationen gemacht werden. Diese muss auch für die Nagelarthrodesen in gleicher gründlicher Technik erfolgen wie für andere Osteosyntheseverfahren.
Die Indikationsstellung der retrograden tibiotalokalkanearen Arthrodese fordert eine Destruktion des oberen und zugleich des unteren Sprunggelenkes. Gesunde Gelenkkompartimente sollen aus Sicht der Autoren mit der retrograden $\mathrm{Na}-$ gelarthrodese nicht übernagelt werden.

Moderne Nagelsysteme wie der T2Sprunggelenkarthrodesenagel (Fa. Stryker) bieten eine anatomische Anordnung der Verriegelungsoption und eine physiologische Valguskrümmung im Rückfuß- 


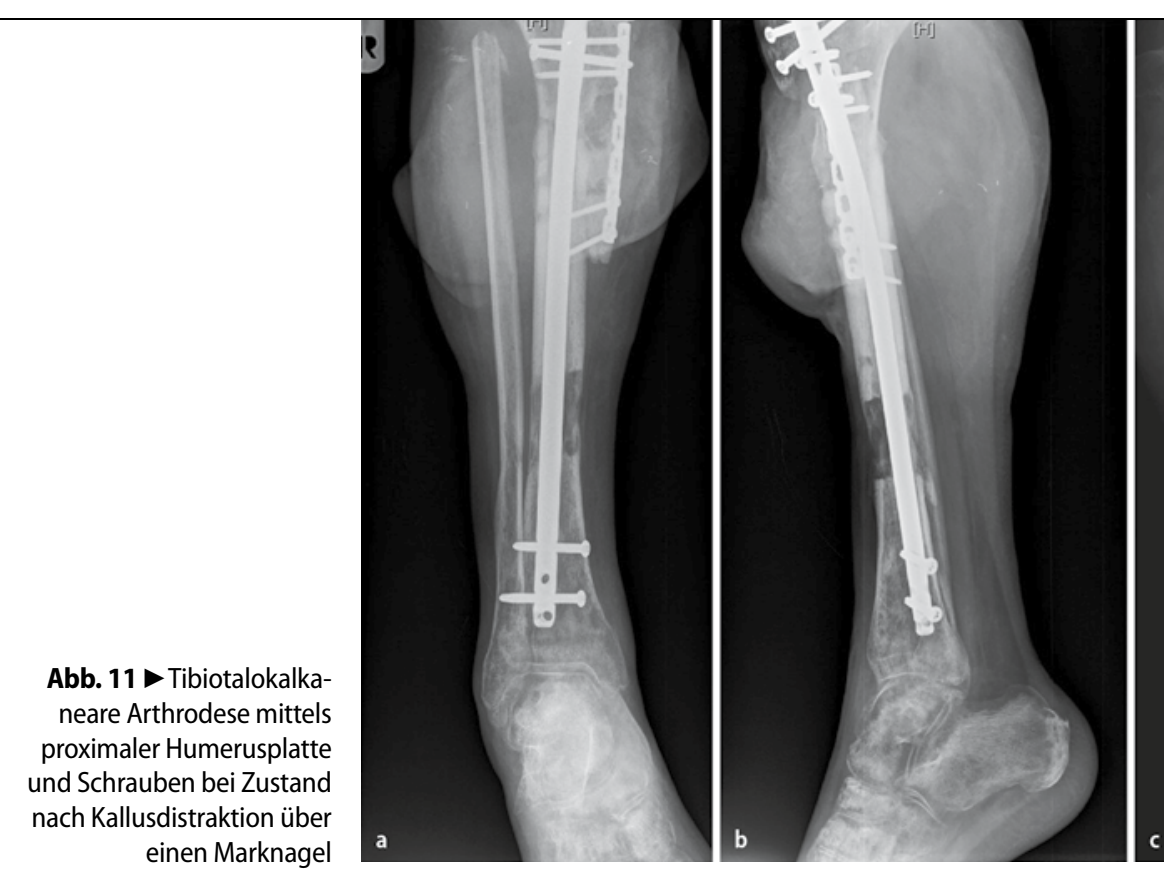

bereich [12]. Die Verriegelungen in diesem Areal sind in der Regel winkelstabil verblockbar, zudem offerieren verschiedene Nagelsysteme Kompressionsoptionen.

Hervorzuheben ist das Potenzial der retrograden Nägel bei Korrekturen von Fehlstellungen. Bei korrekter Platzierung des Nageleintrittsportals in Kalkaneus und Talus und exakter Aufbohrung des Marknagelkanals in der distalen Tibia ist nach Einbringen des Marknagels eine physiologische Achsausrichtung gegeben (• Abb. 1d-g).

\section{Plattentechniken}

Sie werden für Sprunggelenkarthrodesen insgesamt eher selten angewendet. Es gibt die Möglichkeit der ventralen Plattenosteosynthese von tibiotalaren Arthrodesen mit entsprechend angeformten winkelstabilen Platten. Wir verwenden hierzu 3,5-mm-LCP-Radiusplatten (Fa. Synthes, LCP: „locking compression plate“) (- Abb. 10). Allerdings kommen bei uns Plattentechniken am oberen Sprunggelenk nur dann zum Einsatz, wenn im Rahmen von Revisionsarthrodesen durch die isolierte Schraubentechnik nicht genug Stabilität erzielt wird. In diesen Fällen wird die Schraubenarthrodese zusätzlich mit einer ventralen winkelstabilen Platte augmentiert.

Für die tibiotalokalkaneare Arthrodese sind vereinzelt Publikationen über Platten- osteosynthesen vorhanden. Insgesamt nachteilig für dieses Verfahren in diesem inzisionen. Groß auftragende Plattenosteosynthesen sind aufgrund der geringen Weichteilumfänge und der niedrigen Elasbetrachten.

Für Einzelindikation werden von uns winkelstabile proximale Humerusplatten zur Augmentierung kanülierter Schrauben verwendet ( $\bullet$ Abb. 11). Diese Technik wurde bereits biomechanisch [3] getestet und in klinischen Einzelfällen publiziert [1].

\section{Ergebnisse}

Mit den geschilderten aktuellen Techniken der tibiotalaren Arthrodese sind gute Ergebnisse mit einem entsprechendem Zugewinn der Scorewerte und hohen Fusionsraten von bis $\mathrm{zu} 99 \% \mathrm{zu}$ erreichen [16]. Besondere Bedeutung hat die korrekte Einstellung des Rückfußes mit Reorientierung des Talus.

Für die tibiotalokalkanearen Arthrodesen sind niedrigere Fusionsraten anzusetzen als für die isolierten tibiotalaren Arthrodesen. Die Ergebnisse der Scores fallen im Vergleich zur OSG-Arthrodese wie erwartet schlechter aus, und es ist aufgrund der komplexeren Fälle mit mehr Komplikationen zu rechnen [7, 8]. Dennoch zeigen die aktuellen Ergebnisse Bereich sind die notwendigen Weichteiltizität in diesem Bereich meist kritisch zu mit modernen Nagelsystemen gute Fusions- und kalkulierbare Komplikationsraten $[7,8]$. Sie erzielen signifikante Verbesserungen bezüglich Scores und Aspekten der Lebensqualität $[7,8]$.

\section{Diskussion}

Die Indikationen für die Arthrodese im Sprunggelenkbereich (tibiotalar, talokalkanear, tibiotalokalkanear) sind trotz der Fortschritte in der OSG-Prothetik weiter vorhanden und werden aufgrund des demographischen Wandels und der zunehmenden Zahl an Diabetespatienten in den nächsten Jahrzehnten wahrscheinlich ansteigen.

Die Arthrodese der Sprunggelenke erfordert eine dezidierte Diagnostik mit subtiler Untersuchung und entsprechender radiologischer Befundung. Häufig sind neben den Sprunggelenkröntgenaufnahmen in 2 Ebenen eine Standaufnahme des Rückfußes unter Belastung und eine Computertomographie zur exakten Analyse der Fehlstellung und von möglicherweise vorliegenden Knochendefekten erforderlich. Bei unklarer Durchblutungssituation des Taluskorpus ist eine MRT-Untersuchung indiziert. Infiltrationen isoliert des oberen bzw. des unteren Sprunggelenks können zur Differenzierung der Schmerzursache beitragen.

Die Wahl des Zugangs und die Osteosynthesetechnik hängen sehr stark von der 
individuellen Weichteilsituation im Operationsbereich, den vorliegenden Narbenausbildungen und den knöchernen Gegebenheiten ab. Der laterale Zugang ist bei ausgeprägter langjähriger Valgusfehlstellung im Rückfußbereich kritisch zu bewerten, dorsale Zugänge werden in dieser Situation bevorzugt. Die korrekte Einstellung des Rückfußes ist für das funktionelle Outcome entscheidend. Die Osteosynthesetechnik ist für jeden Patienten in Abhängigkeit von der vorliegenden knöchernen Situation individuell zu planen. Die Indikationsstellung für eine autologe Spongiosaplastik ist großzügig zu handhaben. Die Beweglichkeit der angrenzenden Gelenke ist für das funktionelle Outcome wichtig und dementsprechend in der präoperativen Diagnostik zu berücksichtigen. Komplexe Fälle, wie ausgeprägte posttraumatische Deformitäten und Talusnekrosen, sollten aus Sicht der Autoren zur Behandlung in entsprechende Zentren verlegt werden.

\section{Korrespondenzadresse \\ PD Dr. T. Mückley}

Klinik für Unfall-, Hand- und Wiederherstellungschirurgie, Friedrich-Schiller-Universität Jena, Erlanger Allee 101, 07747 Jena

Thomas.Mueckley@med.uni-jena.de

Interessenkonflikt. Keine Angaben

\section{Literatur}

1. Ahmad J, Pour AE, Raikin SM (2007) The modified use of a proximal humeral locking plate for tibiotalocalcaneal arthrodesis. Foot Ankle Int 28:977-983

2. Albert E (1879) Zur Resektion des Kniegelenkes. Wien Med Press 20:705-708

3. Chodos MD, Parks BG, Schon LC et al (2008) Blade plate compared with locking plate for tibiotalocalcaneal arthrodesis: a cadaver study. Foot Ankle Int 29:219-224

4. Elftman $H$ (1960) The transverse tarsal joint and its control. Clin Orthop 16:41-46

5. Glanzmann MC, Sanhueza-Hernandez R (2007) Arthroscopic subtalar arthrodesis for symptomatic osteoarthritis of the hindfoot: a prospective study of 41 cases. Foot Ankle Int 28:2-7

6. Gougoulias NE, Agathangelidis FG, Parsons SW (2007) Arthroscopic ankle arthrodesis. Foot Ankle Int 28:695-706

7. Klos K, Drechsel T, Gras F et al (2009) The use of a retrograde fixed-angle intramedullary nail for tibiocalcaneal arthrodesis after severe loss of the talus. Strategies Trauma Limb Reconstr Sep 10. [Epub ahead of print]
8. Klos K, Drechsel T, Gras F et al (2009) Tibiotalocalcaneal arthrodesis using a compressive retrograde locking nail with hindfoot valgus. Z Orthop Unfall 147:445-451

9. Muckley T, Schutz T, Srivastava S et al (2003) Ankle arthrodesis with intramedullary compression nailing. Unfallchirurg 106:732-740

10. Muckley T, Hofmann G, Buhren V (2005) Tibiotalar arthrodesis with the tibial compression nail. Oper Orthop Traumatol 17:407-425

11. Muckley T, Eichhorn S, Steinhauser E et al (2006) Tibiotalar and tibiotalocalcanear arthrodesis with intramedullary nailing. Biomaterialien 106(9):90-97

12. Muckley T, Ullm S, Petrovitch A et al (2007) Comparison of two intramedullary nails for tibiotalocalcaneal fusion: anatomic and radiographic considerations. Foot Ankle Int 28:605-613

13. O'Neill PJ, Logel KJ, Parks BG et al (2008) Rigidity comparison of locking plate and intramedullary fixation for tibiotalocalcaneal arthrodesis. Foot Ankle Int 29:581-586

14. Pagenstert GI, Barg A, Leumann AG et al (2009) SPECT-CT imaging in degenerative joint disease of the foot and ankle. J Bone Joint Surg Br 91:11911196

15. Santangelo JR, Glisson RR, Garras DN et al (2008) Tibiotalocalcaneal arthrodesis: a biomechanical comparision of multiplanar external fixation with intramedullary fixation. Foot Ankle Int 29:936-941

16. Zwipp H, Rammelt S, Endres T et al (2010) High union rates and function scores at midterm followup with ankle arthrodesis using a four screw technique. Clin Orthop 468:958-968 